\title{
Données probantes en matière d'utilisation optimale des intervalles de dépistage du VIH chez les personnes séronégatives provenant de divers groupes à risque : un protocole d'examen systématique
}

\author{
GP Traversy', T Austin ${ }^{1}$, J Yau'1, K Timmerman ${ }^{1 *}$
}

\section{Résumé}

Contexte : Les recommandations fondées sur des données probantes pour les tests de dépistage du VIH sont essentielles pour les fournisseurs de soins de santé. Cependant, on ne sait pas avec certitude s'il y a suffisamment de données permettant d'appuyer les recommandations relatives aux fréquences de dépistage du VIH dans une variété de groupes à risque d'infection.

Objectif : L'objectif de ce document consiste à décrire le protocole méthodologique d'un examen systématique permettant de recueillir des données probantes pour déterminer la fréquence optimale de dépistage du VIH chez des personnes de divers groupes à risque d'infection pour ce qui est des résultats au niveau des individus et de la santé publique, de même que le rapport coût-avantage.

Méthodologie : Ce protocole respecte les éléments de déclaration PRISMA-P et l'examen est enregistré dans PROSPERO. La population cible comprend des personnes qui pourraient avoir contracté une infection au VIH n'ayant pas été diagnostiquée. Différentes fréquences de dépistage du VIH seront comparées, et les résultats au niveau des individus et de la santé publique, les valeurs/préférences des patients ainsi que les coûts seront évalués. La stratégie de recherche comprendra des recherches dans MEDLINE/Pubmed, Scopus, Embase, Cochrane, PsychINFO et EconLit, de même que des sources de "littérature grise ». Les articles seront triés par titre/résumé et, par la suite, en texte intégral, en double. L'extraction des données pertinentes à partir des références triées sera réalisée par un premier examinateur, puis vérifiée par un second. Plusieurs outils d'évaluation critique seront utilisés pour juger de la qualité des études, et l'approche GRADE servira à apprécier la qualité générale des données probantes. Les données seront synthétisées de manière descriptive et les résultats seront publiés dans une revue examinée par les pairs.

Discussion : Conçu à l'aide d'une importante contribution de la part d'experts en contenu, cet examen systématique aidera à déterminer les principales données probantes en vue d'élaborer des recommandations sur la fréquence du dépistage du VIH.

\begin{abstract}
Affiliation
${ }^{1}$ Centre de la lutte contre les maladies transmissibles et les infections, Agence de la santé publique du Canada, Ottawa (Ontario)
\end{abstract}

*Correspondance : karen. timmerman@phac-aspc.gc.ca

Citation proposée : Traversy GP, Austin T, Yau J, Timmerman K. Données probantes en matière d'utilisation optimale des intervalles de dépistage du VIH chez les personnes séronégatives provenant de divers groupes à risque : un protocole d'examen systématique. Relevé des maladies transmissibles au Canada.

2017;43(2):42-53. https://doi.org/10.14745/ccdr.v43i02a02f

\section{Contexte}

Élément clé du parcours de soins liés au VIH, le dépistage du VIH représente le premier « 90 » de la stratégie mondiale 90-90-90 pour la lutte contre le $\mathrm{VIH} /$ sida du Programme commun des Nations Unies sur le VIH/sida (ONUSIDA) (1). Le diagnostic du $\mathrm{VIH}$ est essentiel pour faire le pont entre les soins et l'instauration d'un traitement antirétroviral, ce qui pourrait entraîner par la suite une diminution de la charge virale, une réduction de l'infectivité et une amélioration des résultats individuels en matière de santé $(2,3)$. Les faibles taux de dépistage et de diagnostic constituent donc un facteur limitant possible de réussite des stratégies de prévention du VIH. Jusqu'à $50 \%$ des nouvelles infections au VIH peuvent être attribuées à des personnes qui ne savent pas qu'elles sont infectées (4-7). Au Canada, on estime que les personnes qui ne sont pas au courant 
de leur infection représentent $21 \%$ de tous les cas d'infection au $\mathrm{VIH}(8)$.

Certaines populations exposées à un risque plus élevé d'infection au VIH, notamment les hommes ayant des relations sexuelles avec d'autres hommes (HARSAH), les utilisateurs de drogues injectables (UDI) ou les membres des peuples autochtones, pourraient tirer profit de tests de dépistage du $\mathrm{VIH}$ plus fréquents. Ainsi, une des questions importantes à répondre en vue de l'élaboration des recommandations et des stratégies pour le dépistage du VIH est la suivante : À quelle fréquence les personnes provenant de différents groupes à risque devraient-elles être soumises à un test de dépistage du $\mathrm{VIH}$ ? La réponse à cette question exige un équilibre entre les avantages potentiels du dépistage accru et l'augmentation des coûts du dépistage, ainsi que la prise en compte des valeurs et des préférences des patients.

Des directives claires et spécifiques quant à la fréquence des tests de dépistage de personnes provenant de différents groupes à risque pourraient aider les fournisseurs de soins de santé à améliorer leurs comportements relatifs au dépistage et à normaliser cette pratique. Un examen systématique des lignes directrices a révélé qu'un certain nombre de lignes directrices recommandent un dépistage annuel de certains groupes de personnes, notamment les hommes ayant des relations sexuelles avec d'autres hommes et les utilisateurs de drogues injectables, alors que d'autres mentionnent des données lacunaires sur la fréquence des tests de dépistage du $\mathrm{VIH}$ dans certains groupes, ce qui entraîne un manque d'uniformité dans les directives (9). À l'échelle fédérale, le Guide pour le dépistage et le diagnostic de l'infection par le VIH de l'Agence de la santé publique du Canada affirme que les personnes ayant des pratiques à risque élevé devraient être soumises à un dépistage du VIH au moins une fois par année, mais que les données permettant de formuler des recommandations pour chacun des scénarios sont insuffisantes (10).

Bon nombre des lignes directrices étudiées dans le cadre de l'examen systématique de lignes directrices susmentionné remontent à il y a plusieurs années. En outre, les personnes responsables de l'élaboration des lignes directrices ne décrivent pas toujours les fondements de leurs recommandations (p. ex. examen systématique ou opinion d'experts). Un examen à jour et en profondeur des données scientifiques liées à la fréquence du dépistage du VIH est justifié et sera utile pour mettre au point des directives opportunes et de qualité au Canada et à l'étranger. L'objectif de cet article est de décrire le protocole d'un examen systématique visant à répondre à un certain nombre de questions liées à la fréquence des tests de dépistage du VIH.

\section{Objectif}

L'objectif de l'examen systématique consiste à évaluer les données scientifiques qui appuient différentes fréquences de dépistage du $\mathrm{VIH}$ chez des personnes provenant de différents groupes à risque qui pourrait avoir contracté une infection au $\mathrm{VIH}$ non diagnostiquée.
Voici la question de recherche générale : Quelle est la fréquence optimale de dépistage du VIH chez des personnes de divers groupes à risque d'infection pour ce qui est des résultats au niveau des individus et de la santé publique, de même que le rapport coût-avantage? Voici certaines sous-questions pertinentes :

- En regard des résultats au niveau des individus et de la santé publique, quelle est la fréquence de dépistage ou quel est l'intervalle de dépistage du VIH les plus efficaces chez les personnes dont la séronégativité est inconnue ou dont la séronégativité a déjà été confirmée?

- Quelles sont les valeurs et les préférences des patients en ce qui a trait à la fréquence de dépistage ou de reprise du dépistage du VIH?

- Quels sont les effets néfastes potentiels associés aux différentes fréquences de dépistage du VIH?

- Quelle est la fréquence ou quel est l'intervalle de reprise du dépistage du VIH les plus rentables chez les personnes dont la séronégativité est inconnue ou dont la séronégativité a déjà été confirmée?

\section{Méthodologie}

Avant l'élaboration de ce protocole, une recherche de la portée a été effectuée afin d'aider à en orienter la mise au point et à relever tout travail semblable. Cette recherche de la portée comprenait un examen des listes de références provenant des principales lignes directrices relevées dans le cadre d'un examen systématique des lignes directrices sur le dépistage du VIH (9), de même que des recherches sur le terme " dépistage du VIH » dans les sites Web des organisations/registres qui suivent : Cochrane, le National Institute for Health and Clinical Excellence (NICE), l'Agence canadienne des médicaments et des technologies de la santé (ACMTS), le ministère de la Santé du Royaume-Uni (NHS), la International Resource for Infection Control (iNRIC) et le PROSPERO (International Prospective Register of Systematic Reviews).

Ce protocole d'examen systématique a été conçu conformément aux protocoles PRISMA-P (Preferred Reporting Items for Systematic Review and Meta-Analysis Protocols) (11). Voir l'annexe 1 pour obtenir une liste des éléments de déclaration PRISMA-P.

Les ébauches du protocole ont été examinées par des pairs, soit plusieurs experts dans l'élaboration de lignes directrices relatives aux maladies infectieuses de l'Agence de la santé publique du Canada avant l'enregistrement de l'examen dans PROSPERO (International Prospective Register of Systematic Reviews) http://www.crd.york.ac.uk/PROSPERO; numéro d'inscription CRD42016046575) (12). En outre, un économiste de la santé a été consulté.

\section{Stratégie de recherche}

Une stratégie complète de recherche a été établie en consultation avec une bibliothécaire de recherche. Avant sa mise à exécution, la stratégie de recherche a également été examinée par un pair, soit par une bibliothécaire de recherche externe. La version intégrale de la stratégie de recherche se trouve à I'annexe 2. 
Les bases de données suivantes feront l'objet de recherches:

- MEDLINE/PubMed

- Scopus

- Embase

- Bibliothèque Cochrane

- PsyciNFO

- EconLit

Les sources de littérature grise suivantes feront l'objet de recherches:

- Open Grey

- ClinicalTrials.gov

- Toutes les sources pertinentes de la liste de vérification de l'outil Matière Grise de l'ACMTS (13)

Les stratégies de recherche qui seront utilisées pour interroger les bases de données Open Grey et ClinicalTrials.gov se trouvent à l'annexe 3.

L'outil Matière grise de l'ACMTS correspond à une liste de vérification servant à orienter les recherches sur la littérature grise effectuées en ligne. Cette liste comprend des sites Web d'évaluation des technologies de la santé à l'échelle nationale et internationale, des organismes de réglementation de dispositifs médicaux et de médicaments, des registres d'essais cliniques, des ressources liées à l'économie de la santé, des bases de données sur la prévalence ou l'incidence, ainsi que des sites Web de listes de médicaments (13). Au total, 40 sites Web pertinents ont été relevés par l'équipe de recherche à l'aide de la liste de vérification (les listes de médicaments et les bases de données sur les mises en garde/précautions relatives à des médicaments et sur la surveillance $\mathrm{n}^{\prime}$ ont pas été considérées comme pertinentes en regard de la question de recherche, par exemple). Bon nombre des sites Web de la liste de vérification ne comprennent aucune une option de recherche avancée, de sorte que la grande majorité feront l'objet d'une recherche en employant le terme " VIH », afin d'obtenir le plus vaste éventail de résultats potentiellement pertinents. Les sites Web dotés de fonctions de recherche avancée feront l'objet de recherches en combinant les termes « VIH » et « test » ou " dépistage ».

Des recherches dans la littérature grise seront effectuées par deux membres de l'équipe de recherche de manière indépendante, et tous les articles considérés comme potentiellement pertinents seront ajoutés aux résultats des recherches dans les bases de données à des fins de tri ultérieur.

\section{Gestion des données}

Toutes les références seront téléchargées dans DistillerSR, un logiciel Web sécurisé destiné à la gestion des examens systématiques en ligne (Evidence Partners). Cette plateforme logicielle sera utilisée pour le tri des articles admissibles, l'extraction de données et l'évaluation de la qualité.

\section{Critères d'admissibilité}

\section{Langue}

Les articles publiés en anglais ou en français seront pris en compte aux fins de l'examen.

\section{Méthodologie de l'étude}

Les méthodologies d'étude admissibles varieront en fonction de la sous-question de recherche. Pour l'ensemble des questions de recherche, les autres examens systématiques ne seront pas explicitement exclus. Au lieu, les listes de références seront parcourues afin d'en relever des articles pertinents qui pourraient avoir été omis.

Le tableau 1 décrit les méthodologies d'étude qui seront prises en compte pour chacune des sous-questions de recherche.

\section{Tableau 1 : Méthodologies d'étude admissibles aux fins d'inclusion dans l'examen par sous-question de recherche}

\begin{tabular}{|c|c|}
\hline Sous-question de recherche & $\begin{array}{c}\text { Méthodologies d'étude } \\
\text { admissibles }\end{array}$ \\
\hline $\begin{array}{l}\text { En regard des résultats au } \\
\text { niveau des individus et de la } \\
\text { santé publique, quelle est la } \\
\text { fréquence de dépistage ou } \\
\text { quel est l'intervalle de reprise } \\
\text { du dépistage du VIH les plus } \\
\text { efficaces chez les personnes } \\
\text { dont la séronégativité } \\
\text { est inconnue ou dont la } \\
\text { séronégativité a déjà été } \\
\text { confirmée? }\end{array}$ & $\begin{array}{l}\text { Études quantitatives (en particulier } \\
\text { celles qui sont comparatives) : } \\
\text { - Essais randomisés } \\
\text { contrôlés (ERC) } \\
\text { - Essais contrôlés non randomisés } \\
\text { - Études d'observation } \\
\text { longitudinales (p. ex. études de } \\
\text { cohorte/registre) } \\
\text { - Méthodologies avant/après } \\
\text { (p. ex. séries temporelles } \\
\text { interrompues) } \\
\text { - Études rétrospectives } \\
\text { - Études sur les interventions } \\
\text { - Études transversales } \\
\text { - Études de modélisation }\end{array}$ \\
\hline $\begin{array}{l}\text { Quelles sont les valeurs et les } \\
\text { préférences des patients en } \\
\text { ce qui a trait à la fréquence } \\
\text { de dépistage ou de reprise du } \\
\text { dépistage du VIH? }\end{array}$ & $\begin{array}{l}\text { Études qualitatives } \\
\text { Enquêtes }\end{array}$ \\
\hline $\begin{array}{l}\text { Quels sont les effets néfastes } \\
\text { potentiels associés aux } \\
\text { différentes fréquences de } \\
\text { dépistage du VIH? }\end{array}$ & $\begin{array}{l}\text { Les études quantitatives et } \\
\text { qualitatives seront prises en } \\
\text { compte. }\end{array}$ \\
\hline $\begin{array}{l}\text { Quelle est la fréquence ou } \\
\text { quel est l'intervalle de reprise } \\
\text { du dépistage du VIH les plus } \\
\text { rentables chez les personnes } \\
\text { dont la séronégativité } \\
\text { est inconnue ou dont la } \\
\text { séronégativité a déjà été } \\
\text { confirmée? }\end{array}$ & $\begin{array}{l}\text { Études sur les coûts: } \\
\text { - Études sur le rapport } \\
\text { coût-avantage, la rentabilité, le } \\
\text { rapport coûts-conséquence, le } \\
\text { rapport coûts-utilité, la réduction } \\
\text { des coûts au minimum } \\
\text { - Études de modélisation }\end{array}$ \\
\hline
\end{tabular}

\section{Population}

La population cible comprend des personnes qui pourraient avoir contracté une infection au VIH n'ayant pas été diagnostiquée.

\section{Intervention}

Le dépistage/le diagnostic du VIH effectués à différents intervalles constituent l'intervention d'intérêt. 


\section{Comparaison}

Les effets de l'intervention seront comparés à l'un ou l'autre des éléments suivants :

- Autres interventions

- Soins « normaux " ou " standard » (de la manière définie dans une étude donnée)

- Avant/après les comparaisons

\section{Milieu}

Toute étude menée dans un milieu où des tests de dépistage du VIH pourraient être effectués sera prise en considération.

\section{Critères d'exclusion}

Les articles seront également exclus dans les cas suivants :

- Publication avant I'an 2000

- Commentaires, éditoriaux, lettres au rédacteur en chef

- Lignes directrices/articles sur des politiques/documents sur des politiques

\section{Résultats}

Plusieurs résultats pouvant être pertinents pour chacune des sous-questions de recherche ont été relevés. Toutefois, il est possible que les résultats ne soient pas tous représentés dans les publications, car certains pourraient ne pas avoir été étudiés. Le tableau 2 présente les résultats principaux et secondaires d'intérêt pour chacune des questions de recherche.

\section{Tri/sélection des articles}

Toutes les références obtenues lors de la recherche seront triées en fonction des titres et des résumés après l'élimination des doublons. Les critères d'admissibilité ci-dessus seront utilisés pour déterminer les critères d'inclusion et d'exclusion des articles au stade de tri par titre/résumé. Le tri des titres et des résumés sera réalisé en double par deux examinateurs. Les désaccords seront réglés lors d'une discussion avec un troisième examinateur.

Le tri du texte intégral sera effectué en fonction des titres et des résumés qui répondent aux critères ci-dessus ou, dans les cas qui soulèvent des incertitudes, il faudra déterminer s'ils y satisfont.

À l'étape de tri du texte intégral, les critères d'admissibilité, ainsi que les résultats énumérés ci-dessus, orienteront le processus d'exclusion des articles non pertinents. Le tri du texte intégral sera réalisé en double par deux examinateurs. Les désaccords seront réglés lors d'une discussion avec un troisième examinateur.

Les raisons de l'exclusion seront consignées à chacune des étapes du processus de tri. Les résultats du tri seront présentés dans un diagramme conforme aux recommandations de PRISMA (14).

\section{Extraction des données}

L'extraction des données pertinentes à partir des références triées sera réalisée par un premier examinateur, puis vérifiée par un second, et ce, en vue de réduire les biais ou les erreurs d'extraction. Tout désaccord sera réglé au moyen d'une discussion avec un ou deux autres examinateurs. En cas $d$ 'incertitudes majeures, il est possible de communiquer avec les auteurs des études. Toutes les données pertinentes (p. ex. année de publication, période de collecte des données, population de l'étude, taille des échantillons, lieu, milieu de l'étude, méthodologie de l'étude, interventions, comparaisons, résultats/ critères d'évaluation, score d'évaluation de la qualité, etc.) seront extraites dans des tableaux de données probantes.

Tableau 2 : Résultats potentiels pour chacune des sousquestions de recherche

\begin{tabular}{|c|c|}
\hline Sous-question de recherche & Résultats \\
\hline \multirow[t]{2}{*}{$\begin{array}{l}\text { En regard des résultats au } \\
\text { niveau des individus et de la } \\
\text { santé publique, quelle est la } \\
\text { fréquence de dépistage ou } \\
\text { quel est l'intervalle de reprise } \\
\text { du dépistage du VIH les plus } \\
\text { efficaces chez les personnes dont } \\
\text { la séronégativité est inconnue ou } \\
\text { dont la séronégativité a déjà été } \\
\text { confirmée? }\end{array}$} & $\begin{array}{l}\text { Principaux résultats : } \\
\text { - Délai entre l'exposition/infection au VIH } \\
\text { et le diagnostic } \\
\text { - Numération des lymphocytes CD4 et/ou } \\
\text { charge virale au moment du diagnostic } \\
\text { - Nombre/pourcentage de patients soumis } \\
\text { à des tests de dépistage du VIH } \\
\text { - Nombre de nouveaux diagnostics de VIH } \\
\text { - } \text { Varmi les divers groupes } \\
\text { personion du nombre/pourcentage de } \\
\text { une infection au VIH non diagnostiquée } \\
\text { (modélisation) }\end{array}$ \\
\hline & $\begin{array}{l}\text { Résultats secondaires : } \\
\text { - Nombre de personnes ayant réussi à } \\
\text { établir une relation avec des soins } \\
\text { - Nombre de patients entreprenant un } \\
\text { traitement antirétroviral } \\
\text { - Nombre d'infections au VIH évitées } \\
\text { (modélisation) }\end{array}$ \\
\hline $\begin{array}{l}\text { Quelles sont les valeurs et les } \\
\text { préférences des patients en ce qui } \\
\text { a trait à la fréquence de dépistage } \\
\text { ou de reprise du dépistage du } \\
\text { VIH? }\end{array}$ & $\begin{array}{l}\text { - Niveau d'aisance des patients à l'égard } \\
\text { du test } \\
\text { - Adoption du dépistage du VIH } \\
\text { - Acceptabilité d'une fréquence de } \\
\text { dépistage donnée }\end{array}$ \\
\hline $\begin{array}{l}\text { Quels sont les effets néfastes } \\
\text { potentiels associés aux différentes } \\
\text { fréquences de dépistage du VIH? }\end{array}$ & $\begin{array}{l}\text { - Détresse psychologique ou autres effets } \\
\text { - Jugychosociaux néfastes } \\
\text { - Stigmatisation } \\
\text { - Faux positifs } \\
\text { - Faux négatifs } \\
\text { Remarque : Les études de résultats } \\
\text { portant sur les effets néfastes de nature } \\
\text { psychosociale ou autre en lien avec des } \\
\text { vrais positifs ou des vrais négatifs ne } \\
\text { seront pas incluses. }\end{array}$ \\
\hline $\begin{array}{l}\text { Quelle est la fréquence ou } \\
\text { quel est l'intervalle de reprise } \\
\text { du dépistage du VIH les plus } \\
\text { rentables chez les personnes dont } \\
\text { la séronégativité est inconnue ou } \\
\text { dont la séronégativité a déjà été } \\
\text { confirmée? }\end{array}$ & $\begin{array}{l}\text { - Tous les résultats des types d'étude } \\
\text { pertinents seront pris en considération. }\end{array}$ \\
\hline
\end{tabular}

Les études en double, les études qui se chevauchent ou les études liées qui sont relevées au cours du processus de tri seront traitées en extrayant les données dans un seul formulaire de collecte ou les données séparément et en combinant celles-ci sous la forme d'une seule entrée, conformément au Cochrane Handbook for Systematic Reviews of Interventions (15). 


\section{Évaluation de la qualité}

Chacune des études incluses dans l'examen fera l'objet d'une évaluation de la qualité, de même que l'ensemble des données probantes. Différentes méthodes d'évaluation seront utilisées de manière appropriée en fonction du type d'étude.

Les outils d'évaluation suivants seront utilisés pour chacune des études :

- Risque de biais du centre Cochrane pour les ERC (15)

- Projet de pratique efficace en santé publique - Outil d'évaluation de la qualité pour d'autres études quantitatives (16-18)

- $\quad$ Critical Appraisal Skills Programme (CASP) - Liste de vérification qualitative pour des études qualitatives (19)

Pour l'ensemble, la qualité des données probantes sera évaluée à l'aide de l'approche GRADE (15).

\section{Synthèse des données}

Les données seront synthétisées de manière descriptive. Si le caractère homogène des données probantes est suffisant, une méta-analyse pourrait être envisagée, même si cela est peu probable en raison de la variété des sources de données probantes recherchées.

\section{Analyse de sous-groupe}

La recherche initiale ne ciblera aucun sous-groupe précis. Si de nouvelles données probantes concernant des sous-groupes précis ( $p$. ex. hommes ayant des relations sexuelles avec d'autres hommes, utilisateurs de drogues injectables, membres des peuples autochtones, etc.) deviennent disponibles, les résultats seront ventilés et déclarés de manière descriptive dans des sections distinctes pour chacun des sous-groupes.

\section{Évaluation des méta-biais}

L'évaluation statistique des méta-biais, tels que le biais de publication pour l'ensemble des études (p. ex. test d'Egger), ne sera probablement pas possible en raison de la grande variété des données probantes recherchées et de la probabilité d'inclusion d'une importante proportion d'études comportant des modèles d'observation (20). La possibilité d'un biais de publication doit toutefois être réduite par le recours à une recherche rigoureuse dans la littérature grise, et la possibilité d'un important biais de publication lié aux études d'observation sera prise en compte au moment d'évaluer l'ensemble des données probantes à l'aide de l'approche GRADE (20). La déclaration sélective de résultats sera évaluée dans le cadre d'essais randomisés contrôlés en comparant les résultats déclarés d'études dont les résultats ont été signalés dans la section sur les méthodes du protocole de l'étude. Cela permettra de tenir compte de l'évaluation de la qualité à l'aide de l'outil Risque de biais du centre Cochrane (15).

\section{Modifications}

L'équipe de recherche ne prévoit apporter aucune modification au protocole avant d'effectuer l'examen systématique. Au besoin, cependant, toutes les modifications seront consignées à mesure qu'elles sont apportées et seront inscrites dans le dossier PROSPERO aux fins de cet examen. Des modifications seront également documentées dans la publication définitive.

\section{Diffusion}

Une ébauche des résultats de l'examen systématique sera préparée et soumise aux fins de publication dans une revue examinée par les pairs. Les résultats seront présentés conformément aux lignes directrices PRISMA (Preferred Reporting Items for Systematic Reviews and Meta-Analyses) (14).

\section{Discussion}

Pour autant que nous sachions, il s'agira du premier examen systématique publié portant sur des données probantes appuyant des recommandations relatives à la fréquence du dépistage du VIH. Les données probantes relevées dans le cadre cet examen pourraient se révéler utiles pour mettre à jour des lignes directrices sur le dépistage du VIH au Canada ou pour en établir de nouvelles; des groupes à l'extérieur du Canada pourraient aussi les trouver utiles. L'élaboration de recommandations fondées sur des données probantes spécifiques aidera les fournisseurs de soins de santé à simplifier et à améliorer leurs pratiques en matière de dépistage du $\mathrm{VIH}$. De telles recommandations peuvent également servir aux membres du grand public à prendre en charge leur santé en matière de sexualité.

L'amélioration du dépistage et du diagnostic du VIH permettra de réduire l'importante proportion de personnes infectées au $\mathrm{VIH}$ qui ne savent pas qu'elles le sont. Ce groupe contribue à une proportion considérable de nouveaux cas d'infection au $\mathrm{VIH}$, et certaines données tendent à indiquer que les personnes infectées seront plus susceptibles de prendre les mesures nécessaires pour réduire au minimum le risque de transmission lorsqu'elles prennent connaissance de leur infection $(4,21)$. Le diagnostic du VIH est également la première étape à franchir en vue d'obtenir un traitement et, par conséquent, l'amélioration du dépistage et du diagnostic offrira des avantages au niveau des personnes et de la santé publique.

Les points forts de ce protocole d'examen systématique protocole comprennent l'importante participation d'experts en contenu à l'élaboration des lignes directrices sur le VIH et les infections transmissibles sexuellement ainsi que l'économie de la santé. Une autre force de cet exercice est l'examen par des pairs externes de la stratégie de recherche élaborée par une bibliothécaire de recherche.

Une des limites de l'examen pourrait être l'inclusion éventuelle d'études principalement d'observation, en raison de la difficulté de mener des études expérimentales sur le sujet. Cela pourrait soulever des préoccupations concernant la qualité des données probantes; toutefois, les résultats des évaluations de la qualité seront publiés et, en conséquence, l'évaluation du biais des données probantes sera transparente.

Si cet examen systématique n'arrive pas à trouver des données probantes pour répondre à une ou à plusieurs des questions de recherche, cela sera mentionné dans les résultats. Un résultat " négatif » serait quand même utile pour les responsables de l'élaboration de lignes directrices, car il confirmerait les 
lignes directrices qui mentionnent le manque de données probantes en vue de formuler des recommandations en matière d'intervalles de dépistage (9) et donnerait à penser que de telles recommandations pourraient devoir être faites à l'échelle des territoires, et ce, en fonction de l'opinion d'experts. Ce résultat aiderait également à souligner les lacunes sur le plan des données probantes, ce qui pourrait être utile pour orienter les efforts de recherche futurs.

\section{Remerciements}

Nous tenons à remercier Michèle Sabourin, Kelsey Young, Lisa Pogany, Rachel Bennett, Ulrick Auguste, Cathy Latham-Carmanico, Bakhtiar Anwar, Jun Wu et Margaret Gale-Rowe pour leur rétroaction et leurs commentaires constructifs sur l'ébauche du protocole. Nous aimerions également remercier les bibliothécaires de recherche, Connie Barrowclough et Katherine Merucci, qui ont aidé à concevoir et à réaliser notre recherche systématique, ainsi qu'à Margaret Sampson, pour avoir effectué l'examen par les pairs de la stratégie de recherche.

\section{Contributions des auteurs}

KT est la responsable. GT a rédigé l'ébauche du protocole de l'examen, et tous les auteurs ont grandement contribué à peaufiner la question/les sous-questions de recherche, les critères d'admissibilité et d'inclusion de l'étude, les résultats d'intérêt, les processus de tri, d'extraction et d'évaluation de la qualité, ainsi que la stratégie de recherche (avec la collaboration d'une bibliothécaire de recherche dûment formée). TA, GT et JY rédigeront l'ébauche de l'article. Tous les auteurs liront, fourniront de la rétroaction et approuveront la version définitive de l'article.

\section{Conflit d'intérêts}

Aucun.

\section{Financement}

Cette étude a été appuyée par l'Agence de la santé publique du Canada. Les auteurs n'ont aucune source de financement extérieure à déclarer.

\section{Références}

1. Joint United Nations Programme on HIV/AIDS. 90-90-90: An ambitious treatment target to help end the AIDS epidemic. Geneva: UNAIDS; 2014.

2. INSIGHT START Study Group, Lundgren JD, Babiker AG, Gordin F, Emery S, Grund B, Sharma S, Avihingsanon A, Cooper DA, Fätkenheuer G, Llibre JM, Molina JM, Munderi P, Schechter M, Wood R, Klingman KL, Collins S, Lane HC,
Phillips AN, Neaton JD. Initiation of antiretroviral therapy in early asymptomatic HIV infection. N Engl J Med. 2015 Aug 27;373(9):795-807.

3. Cohen MS, Chen YQ, McCauley M, Gamble T, Hosseinipour MC, Kumarasamy N, Hakim JG, Kumwenda J, Grinsztejn B, Pilotto JH, Godbole SV, Mehendale S, Chariyalertsak S, Santos BR, Mayer KH, Hoffman IF, Eshleman SH, PiwowarManning E, Wang L, Makhema J, Mills LA, de Bruyn G, Sanne I, Eron J, Gallant J, Havlir D, Swindells S, Ribaudo H, Elharrar V, Burns D, Taha TE, Nielsen-Saines K, Celentano D, Essex M, Fleming TR; HPTN 052 Study Team. Prevention of HIV-1 Infection with early antiretroviral therapy. N Engl J Med. 2011;365(6):493-505.

4. Marks G, Crepaz N, Janssen RS. Estimating sexual transmission of HIV from persons aware and unaware that they are infected with the virus in the USA. AIDS. 2006;20(10):1447-50.

5. McNairy ML, El-Sadr WM. Antiretroviral therapy for the prevention of HIV transmission: what will it take? Clin Infect Dis. 2014;58(7):1003-11.

6. McNairy ML, Cohen M, El-Sadr WM. Antiretroviral therapy for prevention is a combination strategy. Curr HIV/AIDS Rep. 2013;10(2):152-8.

7. Skarbinski J, Rosenberg E, Paz-Bailey G, Hall HI, Rose CE, Viall $\mathrm{AH}$, et al. Human immunodeficiency virus transmission at each step of the care continuum in the United States. JAMA Intern Med. 2015;175(4):588-96.

8. Tomas K, Dhami P, Houston C, Ogunnaike-Cooke S, Rank C. Le VIH au Canada, de 2009 to 2014. Relevé des maladies transmissibles au Canada. 2015;41(12):292. Disponible à l'adresse : http://www.phac-aspc.gc.ca/publicat/ccdrrmtc/15vol41/dr-rm41-12/ar-01-fra.php.

9. Austin T, Traversy GP, Ha S, Timmerman K.

Recommandations canadiennes et internationales sur la fréquence de dépistage et de diagnostic du VIH : Un examen systématique. Relevé des maladies transmissibles au Canada. 2016;42:180-8. Disponible à l'adresse : http:// www.phac-aspc.gc.ca/publicat/ccdr-rmtc/16vol42/dr-rm42-8/ ar-03-fra.php.

10. Agence de la santé publique du Canada. Virus de I'immuniodéficience humaine - Guide pour le dépistage et le diagnostic de l'infection par le VIH. [Internet]. 2012 (mis à jour le 5 août 2014; consulté le 21 nov 2016). Disponible à l'adresse : http://www.phac-aspc.gc.ca/aids-sida/guide/ hivstg-vihgdd-fra.php.

11. Shamseer L, Moher D, Clarke M, Ghersi D, Liberati A, Petticrew M, Shekelle P, Stewart LA; PRISMA-P Group. Preferred reporting items for systematic review and meta-analysis protocols (PRISMA-P) 2015: elaboration and explanation. BMJ. 2015;349:g7647. doi: 10.1136/bmj.g7647.

12. University of York Centre for Reviews and Dissemination. Guidance notes for registering a systematic review protocol with PROSPERO [Internet]. Toronto (ON): Univeristy of York: 2016 May (consulté le 21 nov 2016). Disponible à l'adresse : http://www.crd.york.ac.uk/PROSPERO/documents/ Registering\%20a\%20review\%20on\%20PROSPERO.pdf.

13. Canadian Agency for Drugs and Technologies in Health. Grey Matters: a practical tool for searching healthrelated 
grey literature [Internet]. Ottawa (ON): CADTH; 2015 Dec (consulté oct 2016). Disponible à l'adresse : https://www. cadth.ca/resources/finding-evidence/grey-matters.

14. Liberati A, Altman DG, Tetzlaff J, Mulrow C, Gotzsche PC, loannidis JP, Clarke M, Devereaux PJ, Kleijnen J, Moher D. The PRISMA statement for reporting systematic reviews and meta-analyses of studies that evaluate healthcare interventions: explanation and elaboration. BMJ. 2009;339:b2700. doi: 10.1136/bmj.b2700.

15. Higgins JPT, Green S, editors. Cochrane handbook for systematic reviews of interventions. Version 5.1.0. London (UK): The Cochrane Collaboration; 2011.

16. Armijo-Olivo S, Stiles CR, Hagen NA, Biondo PD, Cummings GG. Assessment of study quality for systematic reviews: a comparison of the Cochrane Collaboration Risk of Bias Tool and the Effective Public Health Practice Project Quality Assessment tool: methodological research. J Eval Clin Pract. 2012;18(1):12-8.

17. Thomas BH, Ciliska D, Dobbins M, Micucci S. A process for systematically reviewing the literature: providing the research evidence for public health nursing interventions. Worldviews Evid Based Nurs. 2004;1(3):176-84.

18. Deeks JJ, Dinnes J, D'Amico R, Sowden AJ, Sakarovitch C, Song F, Petticrew M, Altman DG. Evaluating non-randomised intervention studies. Health Technol Assess. 2003;7(27):1173.

19. Hannes K. Chapter 4: Critical appraisal of qualitative research. In: Noyes J, Booth A, Hannes K, Harden A, Harris J, Lewin S, Lockwood C, editors. Supplementary guidance for inclusion of qualitative research in Cochrane Systematic Reviews of Interventions: Cochrane Collaboration Qualitative Methods Group; 2011.

20. Guyatt GH, Oxman AD, Montori V, Vist G, Kunz R, Brozek J, Alonso-Coello P, Djulbegovic B, Atkins D, Falck-Ytter $Y$, Williams JW Jr, Meerpohl J, Norris SL, Akl EA, Schünemann HJ. GRADE guidelines: 5 . Rating the quality of evidence publication bias. J Clin Epidemiol. 2011:12;64(12):1277-82.

21. Marks G, Crepaz N, Senterfitt JW, Janssen RS. Meta-analysis of high-risk sexual behavior in persons aware and unaware they are infected with HIV in the United States: implications for HIV prevention programs. J Acquir Immune Defic Syndr.

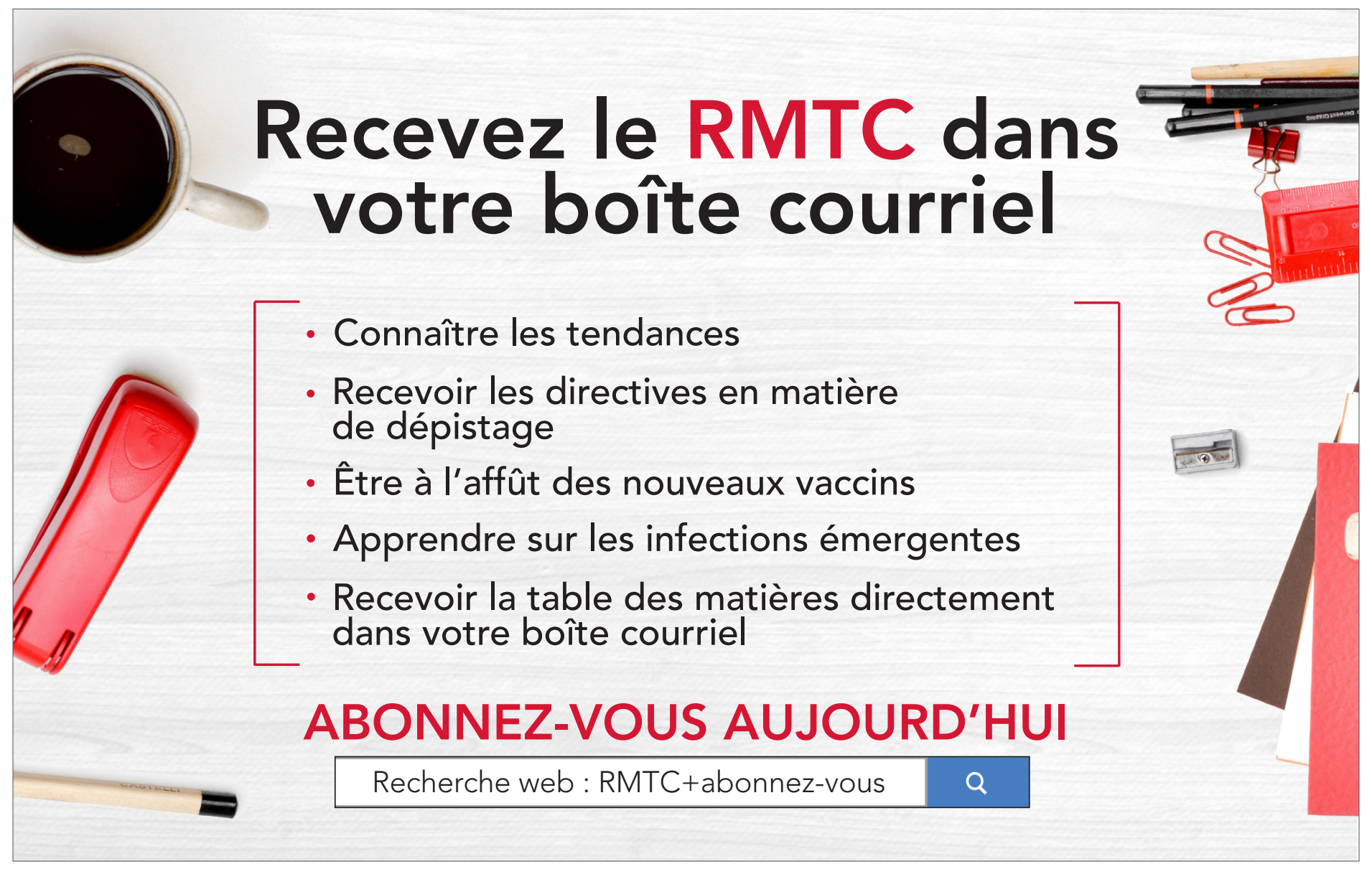




\section{Annexe 1 : Liste de vérification PRISMA-P dûment remplie aux fins de l'examen systématique}

\begin{tabular}{|c|c|c|c|}
\hline Section et sujet & Élément & Élément de la liste de vérification & $\begin{array}{l}\text { No(s) de } \\
\text { la ligne }\end{array}$ \\
\hline \multicolumn{4}{|c|}{ RENSEIGNEMENTS ADMINISTRATIFS } \\
\hline \multicolumn{4}{|l|}{ Titre : } \\
\hline Identification & $1 \mathrm{a}$ & Identifier le rapport en tant qu'un protocole d'un examen systématique & 1,67 \\
\hline Mise à jour & $1 \mathrm{~b}$ & Si le protocole concerne la mise à jour d'un examen systématique antérieur, le mentionner dans le titre & S.O. \\
\hline Inscription & 2 & $\begin{array}{l}\text { Si l'examen est enregistré, fournir le nom du registre (comme PROSPERO) et le numéro } \\
\text { d'enregistrement }\end{array}$ & 103 \\
\hline \multicolumn{4}{|l|}{ Auteurs : } \\
\hline Coordonnées & $3 a$ & $\begin{array}{l}\text { Fournir le nom, l'établissement d'affiliation, I'adresse de courriel de tous les auteurs; fournir l'adresse } \\
\text { postale physique de l'auteur correspondant }\end{array}$ & 4-7 \\
\hline Contributions & $3 b$ & Décrire les contributions des auteurs au protocole et identifier le responsable de l'examen & $262-267$ \\
\hline Modifications & 4 & $\begin{array}{l}\text { Si le protocole représente la modification d'un protocole déjà terminé ou publié, le mentionner } \\
\text { et énumérer les changements; par ailleurs, faire état du plan visant à documenter les importantes } \\
\text { modifications apportées au protocole }\end{array}$ & $220-224$ \\
\hline \multicolumn{4}{|l|}{ Appui : } \\
\hline Sources & $5 a$ & Indiquer les sources de soutien financier ou autre aux fins d'examen & $270-273$ \\
\hline Promoteur & $5 b$ & Fournir le nom du bailleur de fonds et/ou du commanditaire de l'examen & $270-273$ \\
\hline $\begin{array}{l}\text { Rôle du commanditaire } \\
\text { ou du bailleur de fonds }\end{array}$ & $5 c$ & $\begin{array}{l}\text { Décrire les rôles du ou des bailleurs de fonds, commanditaires et/ou établissements, le cas échéant, } \\
\text { dans l'élaboration du protocole }\end{array}$ & S.O. \\
\hline \multicolumn{4}{|l|}{ INTRODUCTION } \\
\hline Justification & 6 & Décrire la justification de l'examen dans le contexte des faits déjà connus & $52-68$ \\
\hline Objectifs & 7 & $\begin{array}{l}\text { Fournir un énoncé explicite de la ou des questions traitées durant l'examen en ce qui a trait aux } \\
\text { participants, aux interventions, aux comparateurs et aux résultats (selon la méthode PICO) }\end{array}$ & $151-174$ \\
\hline \multicolumn{4}{|l|}{ MÉTHODOLOGIES } \\
\hline Critères d'admissibilité & 8 & $\begin{array}{l}\text { Préciser les caractéristiques de l'étude (p. ex. PICO, méthodologie de l'étude, contexte, cadre } \\
\text { temporel) et du rapport (p. ex. années prises en considération, langue, statut de publication) à utiliser à } \\
\text { titre de critères d'admissibilité aux fins de l'examen }\end{array}$ & $140-174$ \\
\hline Sources d'information & 9 & $\begin{array}{l}\text { Décrire toutes les sources d'information (p. ex. bases de données électroniques, coordonnées des } \\
\text { auteurs de l'étude, registres d'essais ou d'autres sources de littérature grise) de même que les dates de } \\
\text { la période couverte prévue }\end{array}$ & $\begin{array}{r}109-129 \\
145,191- \\
192\end{array}$ \\
\hline Stratégie de recherche & 10 & $\begin{array}{l}\text { Présenter une ébauche de stratégie de recherche à utiliser dans au moins une base de données, y } \\
\text { compris les limites prévues de celle-ci, de manière à pouvoir la reproduire }\end{array}$ & 344 \\
\hline \multicolumn{4}{|l|}{ Dossiers de l'étude : } \\
\hline Gestion des données & $11 a$ & $\begin{array}{l}\text { Décrire le ou les mécanismes qui seront utilisés pour gérer les dossiers et les données tout au long de } \\
\text { l'examen }\end{array}$ & 137 \\
\hline Processus de sélection & $11 \mathrm{~b}$ & $\begin{array}{l}\text { Énoncer le processus qui servira à sélectionner les études (p. ex. deux examinateurs indépendants) } \\
\text { dans chacune des phases de l'examen (c'est-à-dire le tri, I'admissibilité et l'inclusion dans la méta- } \\
\text { analyse) }\end{array}$ & $175-187$ \\
\hline $\begin{array}{l}\text { Processus de collecte } \\
\text { des données }\end{array}$ & $11 \mathrm{c}$ & $\begin{array}{l}\text { Décrire la méthode prévue d'extraction des données à partir des rapports ( } p \text {. ex. formulaires pilotes } \\
\text { de manière indépendante et en double) et tout processus visant l'obtention et la confirmation des } \\
\text { données auprès des chercheurs }\end{array}$ & 188-199 \\
\hline Éléments de données & 12 & $\begin{array}{l}\text { Énumérer et définir toutes les variables pour lesquelles des données sont recherchées (p. ex. } \\
\text { éléments PICO, sources de financement), toute hypothèse et simplification prédéterminées en regard } \\
\text { des données }\end{array}$ & 192-194 \\
\hline $\begin{array}{l}\text { Résultats et } \\
\text { priorisation }\end{array}$ & 13 & $\begin{array}{l}\text { Énumérer et définir tous les résultats pour lesquels des données seront recherchées, y compris la } \\
\text { priorisation des résultats principaux et autres, ainsi qu'une justification }\end{array}$ & 173 \\
\hline $\begin{array}{l}\text { Risque de biais dans } \\
\text { chaque étude }\end{array}$ & 14 & $\begin{array}{l}\text { Décrire les méthodes prévues pour évaluer le risque de biais de chacune des études, notamment si } \\
\text { l'évaluation se fera au niveau des résultats ou de l'étude, ou des deux; énoncer la manière dont ces } \\
\text { renseignements seront utilisés dans le cadre de la synthèse des données }\end{array}$ & $200-209$ \\
\hline \multirow[t]{4}{*}{ Synthèse des données } & $15 a$ & Décrire les critères en vertu desquels les données de l'étude seront synthétisées quantitativement & 213 \\
\hline & $15 b$ & $\begin{array}{l}\text { Si les données sont appropriées pour réaliser une synthèse quantitative, décrire les mesures sommaires } \\
\text { prévues, les méthodes utilisées pour le traitement des données et la combinaison des données } \\
\text { provenant diverses études, y compris les moyens prévus pour assurer l'uniformité (p. ex. } I^{2}, T \text { de } \\
\text { Kendall) }\end{array}$ & S.O. \\
\hline & $15 c$ & $\begin{array}{l}\text { Décrire toute autre analyse proposée (p. ex. analyses de sensibilité ou de sous-groupes., méta- } \\
\text { régression) }\end{array}$ & 216-219 \\
\hline & $15 d$ & Si la synthèse quantitative n'est pas appropriée, décrire le type de sommaire prévu & 212-219 \\
\hline Méta-biais & 16 & $\begin{array}{l}\text { Préciser toute évaluation prévue des méta-biais (p. ex. biais de publication pour l'ensemble des études, } \\
\text { rapports sélectifs parmi les études) }\end{array}$ & $220-229$ \\
\hline $\begin{array}{l}\text { Confiance dans les } \\
\text { données probantes } \\
\text { cumulatives }\end{array}$ & 17 & Décrire la façon dont la force de l'ensemble des données probantes sera évaluée ( $p$. ex. GRADE) & $210-211$ \\
\hline
\end{tabular}

Abréviations : No(s) de la ligne, numéro(s) de la ligne; S.O., sans objet 


\section{Annexe 2 : Stratégie de recherche}

Toutes les recherches ont été effectuées et téléchargées le 16 septembre 2016.

\section{Base(s) de données : Econlit (de 1886 à août 2016)}

\section{Stratégie de recherche :}

\begin{tabular}{|c|c|c|}
\hline$N^{\circ}$ & Recherches & Résultats \\
\hline 1 & $\begin{array}{l}\text { (hiv or hiv }+ \text { or hiv-1 or hiv- } 2 \text { or hiv1 or hiv2 or } \\
\text { hivaids or (human immun* adj2 virus) or virus de } \\
\text { l'immunodéficience humaine or vih or vih+ or vih1 } \\
\text { or vih2 or vih-1 or vih-2).af. }\end{array}$ & 1713 \\
\hline 2 & $\begin{array}{l}\text { (screen* ou rescreen* ou test ou tests ou testing ou } \\
\text { tested ou retest* ou depistage ou "diagnostic du } \\
\text { VIH").af. }\end{array}$ & 91581 \\
\hline 3 & (freque* ou interval*).af. & 26404 \\
\hline 4 & 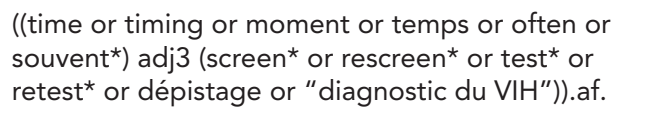 & 1484 \\
\hline 5 & 1 and 2 and 3 & 5 \\
\hline 6 & 1 and 4 & 2 \\
\hline 7 & or/5-6 & 7 \\
\hline 8 & Limit 7 to $y r=" 2000-C u r r e n t "$ & 6 \\
\hline
\end{tabular}

Abréviation : $\mathrm{N}^{\circ}$, numéro
Base(s) de données : PsycINFO de 1806 à la semaine du 4 juillet 2016

Stratégie de recherche :

\begin{tabular}{|c|c|c|}
\hline $\mathrm{N}^{\circ}$ & Recherches & Résultats \\
\hline 1 & $\exp \mathrm{HIV} /$ & 36417 \\
\hline 2 & $\begin{array}{l}\text { (hiv or hiv }+ \text { or hiv-1 or hiv-2 or hiv1 or hiv2 or } \\
\text { hivaids or (human immun* adj2 virus) or virus de } \\
\text { I'immunodéficience humaine or vih or vih+ or vih1 or } \\
\text { vih2 or vih-1 or vih-2).ti. }\end{array}$ & 27738 \\
\hline 3 & $\begin{array}{l}\text { (hiv or hiv }+ \text { or hiv-1 or hiv-2 or hiv1 or hiv2 or } \\
\text { hivaids or (human immun* adj2 virus) or virus de } \\
\text { l'immunodéficience humaine or vih or vih+ or vih1 or } \\
\text { vih2 or vih-1 or vih-2).ab. /freq }=2\end{array}$ & 31713 \\
\hline 4 & or/1-3 & 41632 \\
\hline 5 & screening/ & 8117 \\
\hline 6 & screening tests/ & 5000 \\
\hline 7 & health screening/ & 2451 \\
\hline 8 & $\begin{array}{l}\text { (screen* or rescreen* or test or tests or testing or } \\
\text { tested or retest* or dépistage or "diagnostic du } \\
\text { VIH").ti. }\end{array}$ & 102858 \\
\hline 9 & $\begin{array}{l}\text { (screen* or rescreen* or test or tests or testing or } \\
\text { tested or retest* or dépistage or "diagnostic du } \\
\text { VIH").ab. /freq=2 }\end{array}$ & 258239 \\
\hline 10 & or/5-9 & 304851 \\
\hline 11 & hiv test/ & 1880 \\
\hline 12 & (freque* or interval*).ti. & 22666 \\
\hline 13 & (freque ${ }^{\star}$ or interval*).ab. /freq $=2$ & 91053 \\
\hline 14 & or/12-13 & 100641 \\
\hline 15 & $\begin{array}{l}\text { ((time or timing or moment or temps or often or } \\
\left.\text { souvent }{ }^{\star}\right) \text { adj3 (screen* or rescreen* or test* or } \\
\text { retest }^{\star} \text { or dépistage or "diagnostic du VIH")).ti. }\end{array}$ & 575 \\
\hline 16 & $\begin{array}{l}\text { ((time or timing or moment or temps or often or } \\
\text { souvent*) adj3 (screen* or rescreen* or test* or } \\
\text { retest* or dépistage or "diagnostic du VIH")).ab. / }_{\text {freq }=2}\end{array}$ & 918 \\
\hline 17 & or/15-16 & 1366 \\
\hline 18 & (10 and 14 ) or 17 & 10859 \\
\hline 19 & 4 and 18 & 197 \\
\hline 20 & 11 and (14 or 17 ) & 69 \\
\hline 21 & or/19-20 & 200 \\
\hline 22 & Dissertation Abstract.pt. & 413976 \\
\hline 23 & animals/ & 6443 \\
\hline 24 & case report/ or case report.tw. & 36249 \\
\hline 25 & or/22-24 & 456006 \\
\hline 26 & 21 not 25 & 176 \\
\hline 27 & limit 26 to $y r=" 2000-C u r r e n t "$ & 164 \\
\hline
\end{tabular}

Abréviation : №, numéro 
Bases de données : Epub Ahead of Print, In-Process \& Other Non-Indexed Citations, Ovid MEDLINE(R) Daily et Ovid MEDLINE(R), de 1946 à aujourd'hui (20151216.up)

Stratégie de recherche :

\begin{tabular}{|c|c|c|}
\hline $\mathrm{N}^{\circ}$ & Recherches & Résultats \\
\hline 1 & $\begin{array}{l}\text { (hiv or hiv }+ \text { or hiv-1 or hiv-2 or hiv1 or hiv2 or } \\
\text { hivaids or (human immun* adj2 virus) or virus de } \\
\text { I'immunodéficience humaine or vih or vih+ or } \\
\text { vih1 or vih2 or vih-1 or vih-2).ti,kf. }\end{array}$ & 204027 \\
\hline 2 & $\begin{array}{l}\text { (hiv or hiv }+ \text { or hiv- } 1 \text { or hiv- } 2 \text { or hiv1 or hiv2 or } \\
\text { hivaids or (human immun* adj2 virus) or virus de } \\
\text { l'immunodéficience humaine or vih or vih }+ \text { or } \\
\text { vih1 or vih2 or vih-1 or vih-2).ab. /freq }=2\end{array}$ & 178603 \\
\hline 3 & exp HIV Infections/ & 252533 \\
\hline 4 & $\exp \mathrm{HIV} /$ & 89772 \\
\hline 5 & HIV Antibodies/ & 10284 \\
\hline 6 & or/1-5 & 326372 \\
\hline 7 & mass screening/ & 90016 \\
\hline 8 & Serologic Tests/ & 18348 \\
\hline 9 & $\begin{array}{l}\text { (screen* or rescreen* or test or tests or testing } \\
\text { or tested or retest* or dépistage or "diagnostic } \\
\left.\text { du } \mathrm{VIH}^{\prime \prime}\right) \text {.ti,kf. }\end{array}$ & 470662 \\
\hline 10 & $\begin{array}{l}\text { (screen* or rescreen* or test or tests or testing } \\
\text { or tested or retest* or dépistage or "diagnostic } \\
\text { du } \mathrm{VIH}^{\prime \prime} \text { ).ab. /freq }=2\end{array}$ & 935790 \\
\hline 11 & or/7-10 & 1246845 \\
\hline 12 & AIDS serodiagnosis/ & 6381 \\
\hline 13 & (freque* or interval*).ti,kf. & 132544 \\
\hline 14 & (freque $^{\star}$ or interval ${ }^{\star}$ ).ab. $/$ freq $=2$ & 521701 \\
\hline 15 & or/13-14 & 594802 \\
\hline 16 & $\begin{array}{l}\text { ((time or timing or moment or temps or often or } \\
\left.\text { souvent }{ }^{\star}\right) \text { adj3 (screen* or rescreen* or test or } \\
\text { tests or testing or tested or retest* or dépistage } \\
\left.\text { or "diagnostic du } V\left(\mathrm{H}^{\prime \prime}\right)\right) . t \mathrm{ti}, \mathrm{kf} \text {. }\end{array}$ & 2150 \\
\hline 17 & $\begin{array}{l}\text { ((time or timing or moment or temps or often or } \\
\left.\text { souvent }{ }^{\star}\right) \text { adj3 (screen* or rescreen* or test or } \\
\text { tests or testing or tested or retest* or dépistage } \\
\left.\text { or "diagnostic du } V\left(H^{\prime \prime}\right)\right) . a b . / \text { freq }=2\end{array}$ & 2569 \\
\hline 18 & or/16-17 & 4359 \\
\hline 19 & (11 and 15) or 18 & 54568 \\
\hline 20 & 6 and 19 & 1580 \\
\hline 21 & 12 and (15 or 18$)$ & 157 \\
\hline 22 & 20 not 21 & 1610 \\
\hline 23 & (letter or editorial).pt. & 1360656 \\
\hline 24 & animal/ & 5981714 \\
\hline 25 & human/ & 16333570 \\
\hline 26 & 24 not (24 and 25$)$ & 4284662 \\
\hline 27 & case reports/ or case report.tw. & 1885376 \\
\hline 28 & or/23,26-27 & 7265507 \\
\hline 29 & 22 not 28 & 1567 \\
\hline 30 & limit 29 to $y r=" 2000-C u r r e n t "$ & 1171 \\
\hline
\end{tabular}

Abréviation : $\mathrm{N}^{\circ}$, numéro
Scopus : recherches effectuées le 16 septembre 2016

TITLE ( "hiv" OR "hiv1" OR "hiv2 " OR "hivaids" OR "human immune deficiency virus" OR "human immunodeficiency virus" OR "virus de l'immunodeficience humaine" OR "vih" OR "vih1" OR "vih2" )

AND ( ( TITLE ( ( time OR timing OR moment OR temps OR often OR souvent*) W/3 ( screen* OR rescreen* OR test OR tests OR tested OR testing OR retest* OR "diagnostic du VIH" OR depistage ) ) ) OR ( ( TITLE-ABS ( screen* OR rescreen* OR test OR tests OR tested OR testing OR retest* OR "diagnostic du VIH" OR depistage ))

AND ( TITLE-ABS ( freque* OR interval* ) )) )

AND ( PUBYEAR > 1999 )

AND SUBJAREA ( mult OR arts OR busi OR deci OR econ OR psyc OR soci )

429 dossiers 
Bases de données : Embase, de 1974 au 15 septembre 2016

Stratégie de recherche :

\begin{tabular}{|c|c|c|}
\hline$N^{\circ}$ & Recherches & Résultats \\
\hline 1 & $\begin{array}{l}\text { (hiv or hiv }+ \text { or hiv- } 1 \text { or hiv- } 2 \text { or hiv1 or hiv2 or } \\
\text { hivaids or (human immun* adj2 virus) or virus de } \\
\text { l'immunodeficience humaine or vih or vih }+ \text { or vih1 or } \\
\text { vih2 or vih-1 or vih-2).ti,kw. }\end{array}$ & 246105 \\
\hline 2 & $\begin{array}{l}\text { (hiv or hiv }+ \text { or hiv-1 or hiv- } 2 \text { or hiv1 or hiv2 or } \\
\text { hivaids or (human immun* adj2 virus) or virus de } \\
\text { l'immunodeficience humaine or vih or vih }+ \text { or vih1 or } \\
\text { vih2 or vih-1 or vih-2).ab. /freq }=2\end{array}$ & 204295 \\
\hline 3 & exp Human immunodeficiency virus infection/ & 328710 \\
\hline 4 & exp Human immunodeficiency virus/ & 160405 \\
\hline 5 & Human immunodeficiency virus antigen/ & 1810 \\
\hline 6 & Human immunodeficiency virus antibody/ & 8917 \\
\hline 7 & Human immunodeficiency virus infected patient/ & 26547 \\
\hline 8 & or/1-7 & 435387 \\
\hline 9 & mass screening/ & 52549 \\
\hline 10 & screening/ & 149792 \\
\hline 11 & screening test/ & 55924 \\
\hline 12 & rescreening/ & 227 \\
\hline 13 & serodiagnosis/ & 42942 \\
\hline 14 & $\begin{array}{l}\text { (screen* or rescreen* or test or tests or testing or } \\
\text { tested or retest* or depistage or "diagnostic du } \mathrm{VIH}^{\star} \text { ). } \\
\text { ti,kw. }\end{array}$ & 608987 \\
\hline 15 & $\begin{array}{l}\text { (screen* or rescreen* or test or tests or testing or } \\
\text { tested or retest* or depistage or "diagnostic du } \mathrm{VIH} \text { "). } \\
\text { ab. /freq }=2\end{array}$ & 1265723 \\
\hline 16 & or/9-15 & 1709594 \\
\hline 17 & exp hiv test/ & 6818 \\
\hline 18 & 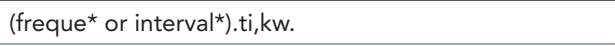 & 162041 \\
\hline 19 & (freque* $^{\star}$ or interval*).ab. /freq $=2$ & 651528 \\
\hline 20 & or/18-19 & 739551 \\
\hline 21 & $\begin{array}{l}\text { ((time or timing or moment or temps or often or } \\
\text { souvent*) adj3 (screen* or rescreen* or test or tests or } \\
\text { testing or tested or retest* or depistage or "diagnostic } \\
\text { du VIH")).ti,kw. }\end{array}$ & 2617 \\
\hline 22 & $\begin{array}{l}\text { ((time or timing or moment or temps or often or } \\
\left.\text { souvent }{ }^{\star}\right) \text { adj3 (screen* or rescreen* or test or tests or } \\
\text { testing or tested or retest* or depistage or "diagnostic } \\
\text { du VIH")).ab. /freq }=2\end{array}$ & 3598 \\
\hline 23 & or/21-22 & 5775 \\
\hline 24 & (16 and 20$)$ or 23 & 81929 \\
\hline 25 & 8 and 24 & 2539 \\
\hline 26 & 17 and (20 or 23 ) & 316 \\
\hline 27 & or/25-26 & 2607 \\
\hline 28 & (letter or editorial).pt. & 1480511 \\
\hline 29 & animal/ & 1803158 \\
\hline 30 & human/ & 17470047 \\
\hline 31 & 29 not (29 and 30$)$ & 1350042 \\
\hline 32 & case study/ or case report.tw. & 362306 \\
\hline 33 & or/28,31-32 & 3168665 \\
\hline 34 & 27 not 33 & 2557 \\
\hline 35 & limit 34 to $y r=" 2000$-Current" & 2174 \\
\hline
\end{tabular}

\section{Cochrane}

Date de la recherche : 16 juin 2016 à 19:05:13.171

\section{Description :}

\begin{tabular}{|c|c|c|}
\hline ID & Résultats & $\begin{array}{l}\text { Nombre } \\
\text { de visites }\end{array}$ \\
\hline$N^{0} 1$ & [mh "HIV Infections"] or [mh HIV] & 9272 \\
\hline$N^{\circ} 2$ & [mh ^"HIV Antibodies"] & 238 \\
\hline$N^{\circ} 3$ & $\begin{array}{l}\text { (hiv or hiv-1 or hiv- } 2 \text { or hiv1 or hiv2 or hivaids } \\
\text { or human immune deficiency virus or human } \\
\text { immunodeficiency virus or human immuno- } \\
\text { deficiency virus or human immune-deficiency } \\
\text { virus or virus de l'immunodéficience humaine } \\
\text { or vih or vih1 or vih2 or vih-1 or vih-2):ti }\end{array}$ & 9923 \\
\hline$N^{\circ} 4$ & $N^{\circ} 1$ or $n^{\circ} 2$ or $n^{\circ} 3$ & 12575 \\
\hline$N^{\circ} 5$ & $\begin{array}{l}{\left[\mathrm{mh} \wedge \wedge^{\prime \prime m a s s ~ s c r e e n i n g "}\right] \text { or }[\mathrm{mh} \wedge \text { "serologic }} \\
\text { tests"] }\end{array}$ & 4877 \\
\hline$N^{\circ} 6$ & $\begin{array}{l}\text { (screen* or rescreen* or test or tests or } \\
\text { tested or testing or retest* or dépistage or } \\
\text { "diagnostic du VII"):ti }\end{array}$ & 21763 \\
\hline $\mathrm{N}^{\circ} 7$ & $N^{\circ} 5$ or $n^{\circ} 6$ & 22979 \\
\hline $\mathrm{N}^{\circ} 8$ & (freque ${ }^{\star}$ or interval*):ti & 6568 \\
\hline$N^{\circ} 9$ & $\begin{array}{l}\text { ((time or timing or moment or temps or } \\
\left.\text { souvent }{ }^{\star}\right) \text { near/3 (screen* or rescreen* or } \\
\text { test or tests or tested or testing or retest* or } \\
\text { "diagnostic du VIH" or dépistage)):ti }\end{array}$ & 147 \\
\hline$N^{\circ} 10$ & $N^{\circ} 8$ or $n^{\circ} 9$ & 6710 \\
\hline$N^{\circ} 11$ & [mh ^ "AIDS serodiagnosis"] & 143 \\
\hline$N^{\circ} 12$ & $\left(n^{\circ} 7\right.$ and $\left.n^{\circ} 8\right)$ or $n^{\circ} 9$ & 332 \\
\hline$N^{\circ} 13$ & $N^{\circ} 10$ and $n^{\circ} 11$ & 3 \\
\hline$N^{\circ} 14$ & $\mathrm{~N}^{\circ} 4$ and $\mathrm{n}^{\circ} 12$ & 17 \\
\hline$N^{\circ} 15$ & $\begin{array}{l}N^{\circ} 13 \text { or } n^{\circ} 14 \text { Publication Year from } 2000 \text { to } \\
2016\end{array}$ & 3 \\
\hline
\end{tabular}




\section{Annexe 3 : Recherche dans la littérature grise}

Toutes les recherches dans la littérature grise ont été effectuées le 27 octobre 2016.

\section{Open Grey}

("HIV" OR "HIV+" or "HIV-1" OR "HIV-2" OR "HIV1" OR "HIV2" OR "HIVAIDS" OR ("human immun*" NEAR/2 "virus") OR

"human immunodeficiency virus" OR "human immunodeficiency virus infection") AND ("screening" OR "test*" OR "retest*" OR

"rescreen*" OR "re-test*" OR "re-screen*")

Résultats : 163 réponses

Lien vers les résultats :

http://www.opengrey.eu/search/request?q=\%28\%22HIV\%22+O

$\mathrm{R}+\% 22 \mathrm{HIV} \% 2 \mathrm{~B} \% 22+\mathrm{or}+\% 22 \mathrm{HIV}-1 \% 22+\mathrm{OR}+\% 22 \mathrm{HIV}-2 \% 22+\mathrm{OR}+$ $\% 22 \mathrm{HIV} 1 \% 22+\mathrm{OR}+\% 22 \mathrm{HIV} 2 \% 22+\mathrm{OR}+\% 22 \mathrm{HIVAIDS} \% 22+\mathrm{OR}+\%$ $28 \% 22$ human+immun $\% 22+N E A R \% 2 F 2+\% 22$ virus $\% 22 \% 29+O R$ +\%22human+immunodeficiency+virus\%22+OR+\%22human+im munodeficiency+virus+infection $\% 22 \% 29+A N D+\% 28 \% 22$ screeni ng\%22+OR+\%22test $\% 22+O R+\% 22$ retest $\% 22+O R+\% 22$ rescre en $\% 22+O R+\% 22$ re-test*\%22+OR+\%22re-screen*\%22\%29+

\section{ClinicalTrials.gov}

Recherche avancée :

Conditions: "HIV" OR "HIV+" or "HIV-1" OR "HIV-2" OR "HIV1" OR "HIV2" OR "HIVAIDS" OR "human immununodeficiency virus"

Interventions: "screening" OR "testing" OR "retesting" OR

"rescreening" OR "re-testing" OR "re-screening"

Intervalle temporel : Études reçues à partir du $1^{\text {er }}$ janvier 2000

Résultats : 649 réponse

Link to results: Clinical Trials.gov (https://clinicaltrials.gov/ct2/ results/displayOpt?flds $=a \& f l d s=b \& f l d s=t \& s u b m i t \_f l d \_o p t=o n$ \&=Update+Display\&cond=\%22HIV\%22+OR+\%22HIV\%2B\%2 $2+$ or $+\% 22 \mathrm{HIV}-1 \% 22+\mathrm{OR}+\% 22 \mathrm{HIV}-2 \% 22+\mathrm{OR}+\% 22 \mathrm{HIV} 1 \% 22$ +OR+\%22HIV2\%22+OR+\%22HIVAIDS\%22+OR+\%22human + immununodeficiency+virus $\% 22$ \&intr $=\% 22$ screening $\% 22+\mathrm{O}$ $\mathrm{R}+\% 22$ testing $\% 22+\mathrm{OR}+\% 22$ retesting $\% 22+\mathrm{OR}+\% 22$ rescreen ing $\% 22+\mathrm{OR}+\% 22$ re-testing $\% 22+\mathrm{OR}+\% 22$ re-screening $\% 22 \& \mathrm{r}$ cv_s $=01 \% 2 F 01 \% 2 F 2000 \&$ show_flds $=Y$ ) 